\title{
Uso da bomba de infusão em terapia intensiva: perspectivas da equipe de enfermagem
}

Use of the infusion pump in intensive care: perspectives of the nursing team

Uso de la bomba de infusión en cuidados intensivos: perspectivas del equipo de enfermería

\section{Jessika Oliveira CavalaroI , Nadia Raquel Suzini Camillo"I, João Lucas Campos de Oliveira ${ }^{\text {III, }}$ Kelly Cristina Inoue ${ }^{\mathrm{IV}}$, Andressa Martins Dias Ferreirav ${ }^{\mathrm{v}}$, Laura Misue Matsuda ${ }^{\mathrm{VI}}$}

\begin{abstract}
Resumo: Objetivo: analisar as percepções dos profissionais de enfermagem sobre o uso da Bomba de Infusão no seu cotidiano em Terapia Intensiva. Método: estudo descritivo-exploratório de abordagem qualitativa, realizado com 15 profissionais de enfermagem da Unidade de Terapia Intensiva Adulto de um hospital-escola público do Paraná. A coleta de dados ocorreu em junho/2017, por meio de entrevistas semiestruturadas gravadas, pautadas na questão norteadora: fale-me sobre o uso da bomba de infusão no seu cotidiano de trabalho. Aos dados transcritos, empregou-se a Análise de Conteúdo. Resultados: foram pontuados aspectos positivos e negativos acerca do uso da bomba de infusão, além de sugestões gerais ao seu propósito. Considerações Finais: apesar dos entrevistados perceberem a Bomba de Infusão como viabilizadora da assertividade na infusão volêmica, existem desvantagens relacionadas ao sensor de gotas e alarme. Como sugestões de melhorias, referiam necessidade de otimizar o design da Bomba de Infusão, especialmente a programação de alarmes.
\end{abstract}

Descritores: Tecnologia biomédica; Bombas de infusão; Segurança do Paciente; Equipe de enfermagem; Unidades de terapia intensiva

Abstract: Objective: to analyze the perceptions of nursing professionals about the use of the Infusion Pump in their daily lives in Intensive Care. Method: descriptive-exploratory study, with a qualitative approach, conducted

\footnotetext{
IEnfermeira. Graduada em Enfermagem. Hospital Santa Casa de Maringá, Maringá, Paraná, Brasil, E-mail: jessika.cavalaro@hotmail.com. ORCID: https://orcid.org/0000-0002-4609-217X

IIEnfermeira. Mestre em Enfermagem. Universidade Estadual de Maringá, Maringá, Paraná, Brasil. E-mail: nadiasuzinicamillo@hotmail.com. ORCID: https://orcid.org/0000-0001-5105-7806

IIIEnfermeiro. Doutor em Enfermagem. Docente na Universidade Federal do Rio Grande do Sul, Porto Alegre, Rio Grande do Sul, Brasil. E-mail: joao-lucascampos@hotmail.com. ORCID: https://orcid.org/0000-0002-1822-2360

${ }^{\mathrm{IV}}$ Enfermeira. Doutora em Enfermagem. Hospital Universitário de Maringá, Maringá, Paraná, Brasil. E-mail: kcinoue1981@gmail.com. ORCID: https://orcid.org/0000-0002-7709-9817

vEnfermeira. Mestre em Enfermagem. Docente na Universidade Estadual de Maringá, Maringá, Paraná, Brasil. E-mail: andressam_dias@yahoo.com.br. ORCID: https://orcid.org/0000-0002-8020-9773

${ }^{\mathrm{VI}}$ Enfermeira. Doutora em Enfermagem. Docente na Universidade Estadual de Maringá, Maringá, Paraná5, Brasil. E-mail: lauramisuem@gmail.com. ORCID: https://orcid.org/0000-0002-4280-7203
} 
Uso da bomba de infusão em terapia intensiva: perspectivas da equipe de enfermagem| 2

with 15 nursing professionals working in the Intensive Care Unit for Adults of a public teaching hospital in Paraná. Data collection took place in June/2017, through recorded semi-structured interviews, based on the guiding question: could tell me about the use of the infusion pump in your daily work? As for the transcribed data, Content Analysis was employed. Results: positive and negative aspects were highlighted regarding the use of the infusion pump, besides general suggestions for its purpose. Final considerations: although the interviewees perceive the Infusion Pump as a viable way for the assertiveness in volemic infusion, there are disadvantages related to the drop and alarm sensor. As for suggestions for improvements, it was mentioned the need to optimize the design of the Infusion Pump, especially the alarm programming.

Descriptors: Biomedical Technology; Infusion Pumps; Patient Safety; Nursing, Team; Intensive Care Units

Resumen: Objetivo: analizar las percepciones de los profesionales de enfermería sobre el uso de la Bomba de Infusión en su vivir cotidiano en Cuidados Intensivos. Método: estudio descriptivo-exploratorio con enfoque cualitativo, realizado con 15 profesionales de enfermería de la Unidad de Cuidados Intensivos para Adultos de un hospital público de enseñanza en Paraná. La recopilación de datos ocurrió en junio/2017, mediante entrevistas semiestructuradas grabadas, basadas en la pregunta orientadora: ¿podrías contarme sobre el uso de la bomba de infusión en su trabajo cotidiano? Para los datos transcritos, se empleó el Análisis de Contenido. Resultados: se señalaron aspectos positivos y negativos acerca del uso de la bomba de infusión, además de sugerencias generales para su propósito. Consideraciones finales: aunque los entrevistados perciben que la Bomba de Infusión es viable para la asertividad en la infusión volémica, existen desventajas relacionadas con el sensor de gotas y alarma. Como sugerencias de mejoras, se mencionó la necesidad de perfeccionar el design de la Bomba de Infusión, especialmente la programación de alarmas.

Descriptores: Tecnología Biomédica; Bombas de Infusión; Seguridad del Paciente; Grupo de Enfermería; Unidades de Cuidados Intensivos

\section{Introdução}

Em Unidade de Terapia Intensiva (UTI), a gestão da tecnologia torna o cuidado mais complexo, em razão de que o gerenciamento deve estar acoplado ao conhecimento sobre cada equipamento que integra a assistência intensiva como: ventiladores, bombas de infusão, monitores e máquinas de diálise. ${ }^{1}$ Nesse cenário, o uso inapropriado, seja por falhas da equipe ou do equipamento, podem ocasionar eventos adversos, cuja prevenção é uma das responsabilidades da equipe envolvida, dentre eles, dos profissionais de enfermagem. ${ }^{2}$ Ao exemplo da premissa anterior, pesquisa com enfermeiros da UTI de um hospital universitário do Rio de Janeiro, constatou deslizes, lapsos e enganos, principalmente ao se operar Bombas de Infusão (BI) e Sistemas de Monitorização. ${ }^{3}$ 
3 | Cavalaro JO, Camillo NRS, Oliveira JLC, Inoue KC, Ferreira AMD, Matsuda LM

A BI consiste em um equipamento eletromédico que, por meio de pressão positiva, gera e regula o fluxo de soluções administradas no indivíduo. ${ }^{4}$ Para isso, possui tela de comando e sistema de alarmes, que permitem maior precisão e segurança na infusão de soluções parenterais ou enterais, indicadas durante o tratamento do paciente. ${ }^{4-7} \mathrm{As}$ BI têm como principal função a administração contínua de drogas que possuem o limiar entre sua terapêutica e sua toxicidade muito próximos, que em apresentações de pílulas e/ou injeções simples trariam oscilações entre sua concentração ao longo do tempo no organismo do indivíduo que recebe tal terapia. Portanto seu uso possibilita maior controle de infusão e, com isso, maior eficácia na terapêutica do paciente - garantindo a segurança da administração de medicamentos. ${ }^{5}$

O uso inapropriado da BI se associa à falta de conhecimento do profissional quanto ao seu funcionamento, à deficiência de comunicação entre a equipe multiprofissional e; também, à falta de atenção no momento do preparo e/ou a carga de trabalho excessiva. ${ }^{8-9}$ Nesse sentido, a variedade de fabricantes e modelos de BI são aspectos importantes a serem considerados, pois há variação da interface da bomba (analógica ou digital), forma de controle da vazão (fluxo volumétrico e não-volumétrico), organização do painel de controle e saída de dados e mecanismo de infusão (peristáltico ou peristáltico rotativo), que podem contribuir à indução do erro na operacionalização desse aparato tecnológico. ${ }^{3}$

Alvitra-se, portanto, que a investigação sobre o uso de BI pelos profissionais de enfermagem é cientificamente relevante porque, trata-se o uso de tecnologia no cuidado ao paciente e frente essa atividade é inerente à administração segura de medicamentos que, por sua vez, corresponde ao terceiro desafio global para a segurança do paciente, lançado pela Organização Mundial de Saúde (OMS) em 2017. ${ }^{10}$ Com isso, espera-se que os resultados desta pesquisa contribuam à melhora da qualidade da assistência à saúde e também, subsidiem construção de novos conhecimentos acerca da tema abordado nas faculdades da área da saúde, nos serviços ou na produção de tais equipamentos. 
Uso da bomba de infusão em terapia intensiva: perspectivas da equipe de enfermagem| 4

Dada a importância de se investigar acerca da compreensão dos profissionais sobre o uso da BI, como alternativa ao aperfeiçoamento do cuidado, este estudo se apoia na questão de pesquisa: como os profissionais de enfermagem percebem o uso da BI no seu trabalho em Terapia Intensiva? A fim de sanar tal indagação, este estudo teve como objetivo analisar as percepções dos profissionais de enfermagem sobre o uso da Bomba de Infusão no seu cotidiano em Terapia Intensiva.

\section{Método}

Pesquisa descritivo-exploratória, de abordagem qualitativa, realizada com profissionais de enfermagem atuantes na UTI para adultos (UTI-A) de um hospital público de ensino, referência para média e alta complexidade. A UTI-A em questão possui 8 leitos e uma equipe de enfermagem composta por 17 enfermeiros e 20 técnicos de enfermagem. Em relação às tecnologias disponíveis ao atendimento, a unidade dispõe de recursos requeridos por legislação específica, ${ }^{11}$ inclusive de BI medicamentosa, BI de dieta enteral e bombas de seringa. A BI volumétrica foi o foco deste estudo porque, na instituição em investigação, este é o modelo utilizado.

Foram convidados a participar da pesquisa os trabalhadores de enfermagem, independente da categoria profissional (enfermeiros e técnicos de enfermagem), aqueles que atenderam aos critérios de seleção: atuar na UTI-A por no mínimo um ano e possuir vínculo empregatício estatutário com o hospital.

Os dados foram coletados em junho de 2017, por meio da entrega de uma carta-convite à Chefia de Enfermagem, para apresentação da proposta do estudo. Após obter a autorização institucional, realizou-se levantamento dos participantes elegíveis. Em seguida, os profissionais de enfermagem foram contatados para convite e esclarecimentos sobre a pesquisa e, mediante o 
5 | Cavalaro JO, Camillo NRS, Oliveira JLC, Inoue KC, Ferreira AMD, Matsuda LM

aceite informal prévio, firmou-se o agendamento da coleta de dados, e realizou-se a entrevista semiestruturada.

Os encontros ocorreram de forma individual, em data, horário e local acordado com cada profissional, segundo sua própria indicação. Nesta ocasião, reiterou-se ao participante o objetivo do estudo e o processo de coleta de dados, de forma a se obter o aceite formal do participante, mediante leitura e assinatura do Termo de Consentimento Livre e Esclarecido em duas vias de igual teor.

Ao início de cada encontro, foi solicitado ao participante o preenchimento de um formulário de caracterização sociodemográfica e laboral, procedendo-se à entrevista com gravação de áudio e pautada na questão norteadora: "Fale-me sobre o uso da bomba de infusão no seu cotidiano de trabalho”. O número de entrevistados foi delimitado com base no alcance do propósito da pesquisa. Para isso, utilizou-se como critério a repetição sucessiva do conteúdo emitido (falas) pelos participantes. ${ }^{12}$

As gravações foram transcritas na íntegra, com adequação ortográfica e na totalidade dos relatos emitidos foi aplicado o método Análise de Conteúdo, na modalidade temática, proposto por Bardin, a qual refere que este método consiste em um conjunto de técnicas de análise, realizado por meio de procedimentos sistemáticos e objetivos de descrição de mensagens, que possibilitam a inferência dos conhecimentos velados por meio das mensagens. ${ }^{13}$

$\mathrm{Na}$ apresentação dos resultados, os excertos/falas/verbatins foram editados sem interferir no conteúdo das mesmas, seguidos pela codificação do respectivo participante, a qual ocorreu pela designação da letra “E” - de entrevistado - e numeração arábica sequencial à transcrição das entrevistas (E1, E2, E3, ... E15). Deste estudo participaram oito enfermeiros e sete técnicos de enfermagem, que responderam a um questionário semiestruturado com seis perguntas previamente redigidas pelo autor. Atendeu integralmente às exigências éticas e legais vigentes ${ }^{14}$ e recebeu Parecer favorável no dia 24/01/2017 de $n^{\circ} .3939 / 2016$, emitido pelo Comitê de 
Uso da bomba de infusão em terapia intensiva: perspectivas da equipe de enfermagem| 6

Ética em Pesquisas Envolvendo Seres Humanos da Universidade Estadual de Maringá e CAAE $n^{\circ}$. 64089117.3.0000.0104.

\section{Resultados}

Os participantes tinham a média de idade de 42 anos; predomínio do sexo feminino ( $\mathrm{n}=13)$; tempo de atuação na UTI investigada, de dois anos (mínimo) a 20 anos (máximo) e; escolaridade que variou de nível técnico a doutorado.

Do conteúdo das entrevistas, pode-se destacar os aspectos positivos e negativos sobre o uso de bomba de infusão e as sugestões para otimizar o seu manuseio.

Quanto aos aspectos positivos da bomba de infusão, a mesma foi considerada como recurso indispensável à manutenção da vida dos pacientes, por permitir a infusão controlada de quase todos os medicamentos administrados na UTI-A:

[...] pensar em terapia intensiva sem bomba de infusão fica bem complicado, porque teria que ter todo um controle, são muitas medicações e realmente é uma grande parceira no nosso trabalho(E5).

[...] a bomba de infusão é o nosso material de trabalho, a gente depende dela porque em um paciente crítico é tudo, é preciso infusão contínua [...] mexemos com muitas drogas vasoativas, temos que fazer uma infusão em tempo preciso, gotejamento preciso. Por isso, é uma ferramenta de trabalho indispensável. (E1)

Ao analisar os excertos, também foram mencionados os benefícios proporcionados pela BI quanto à agilidade e precisão, que favorecem a consecução de atividades rotineiras e também, a segurança do paciente:

Você consegue infundir o volume certo, programar o volume certo [...] sabe que vai infundir tudo certinho, consegue ver em quanto tempo vai acabar tal medicação, para já deixar outra preparada. (E7) 
7 | Cavalaro JO, Camillo NRS, Oliveira JLC, Inoue KC, Ferreira AMD, Matsuda LM

[...] em relação à redução do trabalho da enfermagem e, principalmente, na segurança para o paciente, daquilo que está sendo infundido é extremamente importante. Principalmente em uma UTI, onde as medicações precisam ter gotejamento exato e você precisa ter certeza que isso está realmente acontecendo. (E14)

Alguns participantes relataram como seria a rotina da UTI se não fosse possível o uso da bomba de infusão:

Se fosse manual, com certeza corre-se o risco de [o medicamento] ser infundido a mais ou a menos porque, a enfermagem não tem tempo de ficar cuidando com exatidão de todos os pacientes e de todas as medicações [durante a infusão]. (E12)

[...] manualmente seria bem mais complicado, apesar de sabermos que existe variação de bomba para bomba, de acordo com o equipo. Porém, com a certeza de que a chance de erro é bem menor que o controle manual. (E14)

[...] antes tinha que contar as gotas e na prática não era feita a contagem real do gotejamento [...] ia muito no 'achômetro'. Não tinha nenhuma precisão [...] a gente perdia muito como profissional e também perdia tempo para ver se [o gotejamento] estava muito rápido ou muito lento. (E15)

Embora tenham sido apontados aspectos positivos quanto ao uso de BI em UTI, a interação com a tecnologia também é permeada por aspectos negativos, as quais podem representar barreiras ou limitações.

No que se concerne os aspectos negativos observados durante o uso da BI, dentre as dificuldades relacionadas ao seu uso, apreendeu-se que, para alguns participantes, o cabo do sensor de gotas deste equipamento se constitui em obstáculos à organização do box em que o paciente é internado. Além deste aspecto, também foi destacado como fragilidade, o volume do sistema de alarme sonoro. 
Uso da bomba de infusão em terapia intensiva: perspectivas da equipe de enfermagem| 8

No que diz respeito ao cabo de sensor de gotas, destacaram dificuldade de organização física do equipamento próximo ao paciente e de manuseio no sistema de infusão:

Eu não gosto do sensor da bomba [...] ele enrola a vida da gente! É um fio todo enrolado e esse fio, às vezes, enrosca em suportes, nos equipos e tal. Então, a única coisa que eu não gosto é dos sensores da bomba, é muito complicado. (E4)

[...] e outra coisa que eu acho que passou da hora de resolver, são os sensores dela [da bomba de infusão]. São muito ruins, como se fossem cabos de telefone, é horrível! Dificulta para a gente organizar [o box]. Deixa o box bagunçado [...]. Os sensores batem um no outro. (E5)

Ainda em relação ao sensor de gotas, a dificuldade de higienização também foi destacada:

[...] a limpeza, é difícil; o sensor tem reentrâncias [...]. Sensor usado em paciente com KPC, por exemplo, tem que mandar para óxido [esterilização realizada externamente ao hospital], porque não dá para limpar certinho. Parece um cabo de telefone. (E7)

No que tange ao sistema de alarmes disparados pela BI, os participantes consideram que este provoca excesso de ruído na unidade:

O barulho dentro de uma UTI, com muitos pacientes e muitas bombas, às vezes extrapola e a gente sabe que tem estudos [sobre nível de ruído] que dizem que é prejudicial [para a saúde]. (E14)

O som dela [da BI] na UTI deixa a gente maluca de vez em quando, porque não é um som baixo [...] não pode deixar [o alarme] baixo e quando o alarme da bomba toca, geralmente começa uma atrás da outra [...] e você tem que correr porque o bicho é barulhento!(E8)

Ao explanar suas considerações acerca do uso da BI, os depoimentos transitaram entre aspectos positivos e negativos, permeadas por estratégias de otimização para o manuseio deste equipamento. 
9 | Cavalaro JO, Camillo NRS, Oliveira JLC, Inoue KC, Ferreira AMD, Matsuda LM

Por fim, as sugestões de otimização do manuseio da BI se referem às medidas que podem atenuar ou, até mesmo, sanar as dificuldades relacionadas ao sensor de gotas como ao sistema de alarmes.

Apesar das críticas, o cabo do sensor de gotas da bomba de infusão obteve poucas sugestões de melhora e estas, se relacionam ao seu design:

[...] eu mudaria o design da bomba, colocaria um fio [cabo] reto e não em espiral. (E4)

[...] principalmente a [marca], acho muito grande e espaçosa, ainda que possa sobrepô-las [...]. Talvez se elas [as bombas] fossem menores, pois ocupam um grande espaço!(E11).

No que se refere ao sistema de alarmes, os participantes percebem que o incremento de controle de volume do alarme sonoro e a possibilidade de alteração do padrão de som, facilitam o labor da enfermagem em UTI:

Acho que dava para [o alarme] tocar mais baixinho. Poderia tocar e parar [...]. Poderia ter a opção de diminuir o alarme. (E13).

Uma sugestão seria o controle do volume [...] porque, alguns alarmes são muito altos e a bomba não tem esse controle, se tem não descobrimos; acho que poderia ter isso [...] o alarme incomoda, mas é preciso, não dá para tirar [...] mas poderia ter como diminuir. (E15)

Seria melhor se os alarmes de um box fossem diferentes do outro [...] ficaria mais fácil de saber onde a BI está. Então, você teria eficiência maior para localizar a bomba [...]. A gente precisa de mais três ou quatro tipos de sons [de alarme] porque o negócio é sinistro!(E8)

A gente coloca antibiótico e dali meia hora todas as bombas tocam o alarme e se tem uma droga vasoativa ali no meio [da infusão] a gente acaba ignorando ou até perdendo. Então, se tivesse um alarme diferente para a 
Uso da bomba de infusão em terapia intensiva: perspectivas da equipe de enfermagem| 10

droga poderíamos identificar com mais rapidez e atuar mais onde é prioridade. (E15)

Nos depoimentos, não foram constatadas apenas sugestões a respeito dos problemas sinalizados como aspectos negativos durante o uso da bomba de infusão. Os participantes também teceram as seguintes sugestões para melhor funcionalidade do equipamento:

\section{[...] bomba que também infundisse sangue. (E5)}

As bombas que a gente usa para infundir soro, poderiam ter essa questão de programar para funcionar na hora certa sem que você vá até lá e ligue novamente. É deixar programada. (E7)

[...] minha sugestão, apesar de saber que é possível, é modificar a vazão do KVO [Keep Vein Open ou manutenção da veia aberta]. Na prática a gente não faz isso porque precisa mandar para a fábrica [reprogramar], mas seria muito útil se cada um pudesse controlar esse tempo de acordo com o seu local de trabalho, a característica da unidade, etc. (E14)

Em síntese, as sugestões se direcionaram à resolução de problemas vivenciados cotidianamente pelos profissionais de enfermagem da UTI-A.

\section{Discussão}

As bombas de infusão foram fortemente incorporadas à rotina da UTI, principalmente por sua precisão na infusão de drogas e líquidos utilizados no tratamento de pacientes críticos que, devido à sua complexidade e instabilidade vital, demandam maior exatidão na administração de soluções. ${ }^{7}$ Essa premissa foi fortalecida pelas falas dos profissionais de enfermagem ao retratarem aspectos positivos do uso da BI.

Nos excertos apresentados, percebe-se como a BI está diretamente relacionada a questões de segurança do paciente e ao favorecimento das condições de trabalho da enfermagem. Fato que contrasta com um estudo realizado em Centro de Terapia Intensiva de 
um hospital da Rede Brasileira de Hospitais Sentinela, em qual identificou-se manipulação inadequada da BI, ao silenciar o alarme sonoro mesmo quando este alerta para término de solução e demora em responder ao alarme. ${ }^{9}$

Em UTI, existem múltiplas drogas necessárias ao tratamento do paciente grave, em sua maioria por via intravenosa. ${ }^{15}$ Nesse aspecto, foi destacada a necessidade de rigor no controle durante a administração de drogas vasoativas, indicadas para restaurar a circulação sanguínea adequada reforçando a afirmação de que, as consequências de doses incorretas ou excessivas de medicamentos, podem ser catastróficas e até mesmo letais para o paciente. ${ }^{16}$

Outro quesito positivo, correspondeu à possibilidade de previsão do término da solução em infusão o que, certamente, contribui ao processo de planejamento e organização da assistência de enfermagem em UTI, de modo a garantir que drogas de infusão contínua não tenham o seu fluxo interrompido, visto que o cuidado avançado realizado em terapia intensiva não seria possível sem tais equipamentos de alta tecnologia sendo vista como um importante mecanismo tanto quanto a mão de obra da enfermagem. ${ }^{1}$

Não obstante, apesar do uso da BI favorecer o cuidado de enfermagem em UTI, bem como para a segurança do paciente e organização da rotina da equipe de enfermagem, também foi possível evidenciar dificuldades pautadas no uso do referido equipamento, mais especificamente relacionadas aos sensores de gotas e ao disparo de alarmes. Sobre isso, foram sugeridas modificações na BI, que poderiam melhorar o uso deste equipamento na prática clínica.

A principal queixa sobre os cabos dos sensores de gota foi a conformação do cabo extensor, o qual é espiralado, semelhante ao design de cabos telefônicos. Esse formato é justificado pelo fabricante para permitir maior maleabilidade no manuseio e facilidade de acoplamento do componente à câmara de gotejamento do equipo do sistema de infusão. ${ }^{17}$ 
Uso da bomba de infusão em terapia intensiva: perspectivas da equipe de enfermagem| 12

Ainda que a facilidade no manuseio do fio espiralado de conexão da BI seja a intenção do fabricante, esta parece não atender às necessidades dos entrevistados. Além disso, o seu formato do extensor também inviabiliza a desinfecção eficiente, o que pode causar problemas reais, já que microrganismos, inclusive resistentes, contaminam o aparelho e possuem provável relação com a forma como a limpeza é realizada. ${ }^{18}$ Tal problema poderia ser minimizado com o desenvolvimento de sensores com cabos em outros formatos, ou por funcionamento via wireless.

No que tange ao sistema de alarmes, estes são disparados quando a carga da bateria está perto do fim; o gotejamento está ausente e/ou mais lento do que foi programado; entra ar no equipo ou; quando a infusão do medicamento está chegando ao final, dentre outros..$^{6,17}$ Independentemente da causa e especificação visual no display do equipamento, o volume e padrão de som são os mesmos em todas as bombas.

Apesar de a padronização do alarme da BI ser percebida como um problema pela equipe de enfermagem é preciso lembrar que em UTI, existem outros equipamentos que dispõem deste tipo de sistema para auxiliar a equipe na identificação precoce de alterações clínicas e adoção de condutas para o restabelecimento das funções orgânicas. ${ }^{1-2}$ Nesse cenário, os alarmes da infusão de soluções poderiam ser mais facilmente confundidos com disparos sonoros de outros equipamentos, como ventiladores mecânicos e monitores multiparamétricos.

Acresça-se a premissa anterior que, a possibilidade de configuração do volume do alarme, também pode se comportar como fator de risco durante o uso do equipamento porque, de acordo com a literatura, isso ocorre quando se adotam comportamentos de violação pela equipe como, desabilitar o alarme ou reduzir o som em níveis inaudíveis. ${ }^{3,8}$ Desse modo, alvitrase que, ações desse tipo são contraproducentes por se colocar contra a meta da segurança do paciente. ${ }^{10}$

O excesso de ruído ocasionado pelos alarmes incessantes das BI, no entanto, é um problema compartilhado por outras UTI. Ao literatura indica que o volume excessivo dos 
alarmes causa fadiga nos profissionais da equipe, com consequente dessensibilização do trabalhador ao som, redução do seu estado de alerta e, consequentemente, não atendimento à urgência que induz ao alarme. ${ }^{19}$ Além da fadiga nos trabalhadores, o excesso de ruídos ocasionados pela $\mathrm{BI}$, parece repercutir nos pacientes internados em UTI, uma revisão sistemática com foco na exposição à ruídos em unidade intensiva, constou que mesmo sedados/inconscientes, os pacientes são capazes de reconhecer o som da BI. ${ }^{20}$

No que concerne às sugestões dadas pelos participantes, apesar de não ter sido verificado comprometimento do hemocomponente ao se testar três modelos de dispositivos de infusão intravenosa, a regulamentação específica que trata de procedimentos hemoterápicos faz menção apenas às especificações técnicas dos equipos a serem utilizados no ato transfusional. ${ }^{21}$ Cabe ressaltar que, em revisão de literatura realizada em 2015, constatou a ocorrência de hemólise ao administrar hemocomponente em bomba de infusão. ${ }^{22}$

Com relação à alteração da taxa de KVO (estado de manutenção de veia aberta) in loco, este procedimento já pode ser executado por quem maneja a BI e se encontra descrito no manual do equipamento em análise. ${ }^{17}$ Dúvidas ou desconhecimento dessas informações evidenciam a necessidade de treinamento contínuo dos profissionais e verificação quanto à disponibilidade de manuais correspondentes aos equipamentos em uso, com acesso facilitado para leitura e resolução de questões do cotidiano.

O conhecimento superficial da BI volumétrica da marca adotada na instituição pesquisada pode ser justificado pela variedade de bombas presentes na UTI em estudo. Além desta, o setor dispõe de BI específicas para dieta enteral e bombas de seringa, cuja pluralidade de equipamentos com praticamente a mesma finalidade pode influenciar no desconhecimento de funções programáticas que o equipamento eletromédico em questão possa ter.

Por fim, a sugestão pertinente à programação antecipada de infusões a serem iniciadas automaticamente, tem-se conhecimento que este recurso se faz presente em equipamentos de 
Uso da bomba de infusão em terapia intensiva: perspectivas da equipe de enfermagem| 14

outras marcas, diferentes daquela utilizada na UTI em foco. Contudo, é prudente salientar que, apesar da sua importância, a infusão retardada não deve prejudicar os cuidados para manutenção da permeabilidade de acessos vasculares. ${ }^{23}$

Embora os participantes tenham indicado limitações na BI com consequentes sugestões de adequação, há necessidade de aprofundamento no conhecimento técnico e científico que subsidiem as alterações propostas.

\section{Considerações Finais}

É notável a dupla percepção da equipe de enfermagem quanto à presença da BI no cotidiano do cuidado intensivo - em que destacaram os aspectos positivos e negativos acerca do uso de tal equipamento durante o processo do cuidado. Ao pontuar os aspectos positivos, o controle assertivo da infusão volêmica que trás consigo o rigor do tempo de administração, proporcionando exatidão e segurança na administração de medicamentos.

Foram mencionados alguns pontos negativos que esse aparato tecnológico impõe ao cuidado ao paciente de UTI como, dificuldade no seu manejo; imutabilidade do alarme, quanto à sua diferenciação e ao volume; além de referências ao seu design.

A quantidade reduzida de estudos que exploram este assunto trouxe dificuldades para encontrar referências que corroborassem ou se opusessem às falas encontradas nas entrevistas, porém ao mesmo tempo trouxe maior relevância à pesquisa ao considerar sua aplicabilidade no âmbito prático e para servir de base para investigações futuras. Desta forma, ressalta-se a importância deste trabalho para o planejamento de estratégias de inserção e de utilização correta desta tecnologia na assistência em saúde, bem como para indicar possíveis adequações a ser realizadas para melhor funcionalidade do equipamento. 
15 | Cavalaro JO, Camillo NRS, Oliveira JLC, Inoue KC, Ferreira AMD, Matsuda LM

\section{Referências}

1. Tunlind A, Granström J, Engström A. Nursing care in a high-technological environment: experiences of critical care nurses. Intensive Crit Care Nurs [Internet]. 2015 abr [acesso em 2017 out 12];31(2):116-23. Disponível em: https://www.ncbi.nlm.nih.gov/pubmed/25442241 doi: http://dx.doi.org/10.1016/j.iccn.2014.07.005.

2. Ribeiro GSR, Silva RC, Ferreira MA. Technologies in intensive care: causes of adverse events and implications to nursing. Rev Bras Enferm [Internet]. 2016 set-out [acesso em 2017 set 09];69(5):972-80. Disponível em: http:/www.scielo.br/pdf/reben/v69n5/en_0034-7167-reben-69-050972.pdf. doi: http://dx.doi.org/10.1590/0034-7167.2016690505.

3. Ribeiro GSR, Silva RC, Ferreira MA, Silva GR. Slips, lapses and mistakes in the use of equipment by nurses in an intensive care unit. Rev Esc Enferm USP [Internet]. 2016 [acesso em 2017 out 03];50(3):419-26. Disponível em: http://www.scielo.br/pdf/reeusp/v50n3/0080-6234reeusp-50-03-0419.pdf doi: http://dx.doi.org/10.1590/S0080-623420160000400007.

4. Associação Brasileira de Normas Técnicas (ABNT). Norma NBR IEC 60601-2-24. Equipamento eletromédico - Parte 2-24: prescrições particulares para segurança de bombas e controladores de infusão. Rio de Janeiro (RJ): ABNT; 1999.

5. Holsbach LR, Kliemann Neto FJ, Holsabach N. Utilização do instrumento de identificação de conhecimentos para administração segura de medicamentos com o uso de infusão automática. Rev Bras Eng Bioméd [Internet]. 2013 [acesso em 2017 out 22];29(4):353-62. Disponível em: http://www.scielo.br/pdf/rbeb/v29n4/a05v29n4.pdf. doi: http://dx.doi.org/10.4322/rbeb.2013.034.

6. Dumas Junior A. Estudo metrológico volumétrico de bombas de infusão peristálticas lineares [dissertação]. Curitiba (PR): Universidade Tecnológica Federal do Paraná; 2016. 77 p.

7. Souza V, Cortez EA, Carmo TG. Medidas educativas para minimizar os riscos ocupacionais na equipe de enfermagem da UTI. Rev Pesqui Cuid Fundam [Internet]. 2017 abr-jun [acesso em 2017 ago 10];9(2):583-91. Disponível em: http://www.seer.unirio.br/index.php/cuidadofundamental/article/view/4407 doi: http://dx.doi.org/10.9789/2175-5361.2017.v9i2.583-591.

8. Ribeiro GSR, Silva RC, Ferreira MA, Silva GR. Violações no uso de equipamentos por enfermeiros na terapia intensiva. Texto \& Contexto Enferm [Internet]. 2017 jun [acesso em 2017 out 3];26(2):1-9. Disponível em: http://www.scielo.br/pdf/tce/v26n2/pt_0104-0707-tce-26-02e6050015.pdf doi: http://dx.doi.org/10.1590/0104-07072017006050015. 
Uso da bomba de infusão em terapia intensiva: perspectivas da equipe de enfermagem| 16

9. Duarte SCM, Queiroz ABA, Büscher A, Stipp MAC. O erro humano no cotidiano da assistência de enfermagem em terapia intensiva. Rev Latinoam Enferm [Internet]. 2015 novdez [acesso em 2017 set 09];23(6):1074-81. Disponível em: http://www.scielo.br/pdf/rlae/v23n6/pt_0104-1169-rlae-23-06-01074.pdf doi: http://dx.doi.org/10.1590/0104-1169.0479.2651.

10. Organização Mundial da Saúde (OMS). Patient Safety: making health care safer. Genebra (CH): Organização Mundial de Saúde; 2017.

11. Agência Nacional de Vigilância Sanitária (BR). Resolução RDC $\mathrm{n}^{\circ} 7$, de 24 de fevereiro de 2010. Brasília (DF): ANVISA; 2010.

12. Fontanella BJB, Ricas J, Turato ER. Amostragem por saturação em pesquisas qualitativas em saúde: contribuições teóricas. Cad Saúde Pública [Internet]. 2008 [acesso em 2017 ago 10];24(1). Disponível em: http://www.scielo.br/pdf/csp/v24n1/02.pdf doi: http://dx.doi.org/10.1590/S0102-311X2008000100003.

13. Bardin L. Análise de conteúdo. Lisboa: Edições 70; 2011.

14. Conselho Nacional de Saúde (BR). Resolução n 466, de 12 de dezembro de 2012. Brasília (DF): CNS; 2012.

15. Marsilio NR, Silva D, Bueno D. Drug incompatibilities in the adult intensive care unit of a university hospital. Rev Bras Ter Intensiva [Internet]. 2016 abr-jun [acesso em 2017 out 11];28(2): 147-53. Disponível em: http://www.scielo.br/pdf/rbti/v28n2/en_0103-507X-rbti-28-02-0147.pdf doi: http://dx.doi.org/10.5935/0103-507X.20160029.

16. Silva R, Amante LN, Salum NC, Girondi JBR, Sebold LF. Incidentes e eventos adversos no transporte intra-hospitalar em terapia intensiva. Rev Enferm Cent Oeste Min [Internet]. 2018 [acesso em 2020 mar 12];8:e2805. doi: http://dx.doi.org/10.19175/recom.v8i0.2805.

17. Laboratórios B. BRAUN S.A. Manual do usuário válido para software 5.00: Infusomat Compact. B-BRAUN. 2018 [acesso em 2020 abr 03]. Disponível em: https://www.bbraun.com.br/content/dam/catalog/bbraun/bbraunProductCatalog/S/AEM2015/ptbr/b0/instrucao-de-uso7008001rev4.pdf.bb-.63018877/instrucao-de-uso7008001rev4.pdf

18. Araujo MQ, Carvalhaes RP, Faria SM, Marinho MF, Santos FM, Santos TGQA, et al. Perfil de resistência bacteriana em fômites de UTI em hospital público do Estado do Tocantins. Rev Cereus [Internet]. 2017 [acesso em 2020 mar 12];9(2). Disponível em: http://ojs.unirg.edu.br/index.php/1/article/view/1576/546 
17 | Cavalaro JO, Camillo NRS, Oliveira JLC, Inoue KC, Ferreira AMD, Matsuda LM

19. Amorim SC, Souza HV. Síndrome de Burnout em profissionais da enfermagem que atuam na Unidade de Terapia Intensiva. Rev Pró-UniverSUS [Internet]. 2018 [acesso em 2020 mar 15];09(2): 02-05. Disponível

em:

http://editora.universidadedevassouras.edu.br/index.php/RPU/article/view/1267.

20. Sá RBMA, Cruz ICF. Exposure to noise in intensive care: systematic literature review for a clinical protocol. J Specialized Nurs Care [Internet]. 2016 [acesso em 2017 out 12];8(1). Disponível em: http://www.jsncare.uff.br/index.php/jsncare/article/view/2834/696

21. Ministério da Saúde (BR). Portaria $n^{\circ}$ 158, de 4 de fevereiro de 2016. Redefine o regulamento técnico de procedimentos hemoterápicos. Brasília (DF): Ministério da Saúde; 2016 [acesso em 2020 mar 15]. Disponível em: https://bvsms.saude.gov.br/bvs/saudelegis/gm/2016/prt0158_04_02_2016.html

22. Pardo LP, Kusahara DM, Peterlini MAS, Avelar AFM, Pedreira MLG. Hemólise de eritrócitos em dispositivos de infusão intravenosa: revisão integrativa da literatura. Cogitare Enferm [Internet]. 2015 [acesso em 2020 mar 10];20(1):180-90. Disponível em: http://docs.bvsalud.org/biblioref/2016/07/623/37924-151107-1-pb.pdf

23. Agência Nacional de Vigilância Sanitária (BR). Medidas de prevenção de infecção relacionada à Assistência à Saúde. Brasília (DF): ANVISA; 2017. (Série Segurança do Paciente e Qualidade em Serviços de Saúde).

\section{Autor correspondente}

Jessika Oliveira Calavaro

E-mail: jessika.cavalaro@hotmail.com

Endereço: Rua Marcilio Dias, 737, ap 21, Zona 03 - Maringá, Paraná, Brasil

CEP: 87050-120

\section{Contribuições de Autoria}

\section{1 - Jessika Oliveira Cavalaro}

Concepção e planejamento do projeto de pesquisa; obtenção de dados; análise e interpretação dos dados; redação.

\section{2 - Nadia Raquel Suzini Camillo}

Concepção e planejamento do projeto de Pesquisa; análise e interpretação dos dados; redação.

\section{3 - João Lucas Campos de Oliveira}


Uso da bomba de infusão em terapia intensiva: perspectivas da equipe de enfermagem| 18

Concepção e planejamento do projeto de Pesquisa; análise e interpretação dos dados; redação.

\section{4 - Kelly Cristina Inoue}

Obtenção de dados e revisão crítica.

\section{5 - Andressa Martins Dias Ferreira}

Aanálise e interpretação dos dados e redação.

\section{6 - Laura Misue Matsuda}

Concepção e planejamento do projeto de pesquisa, análise e interpretação dos dados, redação e revisão crítica.

\section{Como citar este artigo}

Cavalaro JO, Camillo NRS, Oliveira JLC, Inoue KC, Ferreira AMD, Matsuda LM. Uso da Bomba de infusão em Terapia Intensiva: perspectivas da equipe de enfermagem. Rev. Enferm. UFSM. 2020 [Acesso em: Anos Mês Dia]; vol.10, e32 1-18. DOI:https://doi.org/10.5902/2179769233455 